\title{
A cross-sectional study of sub-clinical Plasmodium falciparum infection in HIV-1 infected and uninfected populations in Mozambique, South-Eastern Africa
}

Emilia V Noormahomed ${ }^{1,2}$, Marika Orlov², Virgilio do Rosario ${ }^{3}$, Brett W Petersen², Carly Guthrie ${ }^{2}$, Roberto Badaro ${ }^{2,4}$ and Robert T Schooley ${ }^{2,5^{*}}$

\begin{abstract}
Background: Plasmodium falciparum and HIV-1 infection cause substantial morbidity and mortality in sub-Saharan Africa. Increasing evidence suggests these two pathogens interact negatively when infecting the same individual.

Methods: A cross-sectional study among HIV-1 infected and uninfected populations was recruited in Mocuba and Maputo, Mozambique to determine the prevalence of sub-clinical malarial parasitaemia using light microscopy and a nested PCR assay.

Results: The prevalence of sub-clinical $P$. falciparum parasitaemia was low in Maputo, whether determined by microscopy (0.4\%) or PCR (1.9\%), but substantially higher in Mocuba (7.6 and 14.7\%, respectively). Nested PCR detected nearly $70 \%$ more cases of sub-clinical parasitaemia than microscopy, but differences occur by locality. HIV-1 infected persons were more likely to be sub-clinically parasitaemic than HIV-1 uninfected individuals recruited from the same geographic areas. Trimethoprim-sulphamethoxazole use did not substantially reduce sub-clinical parasitaemia.

Conclusions: Dried blood spots are a convenient and sensitive technique for detecting sub-clinical infection with $P$. falciparum by nested PCR. Prevalence of $P$. falciparum is substantially lower in Maputo where malaria control programmes have been more active than in the rural town of Mocuba. In Mocuba, among those presenting for HIV-1 counseling and testing, the prevalence of $P$. falciparum is substantially higher in those who test positive for HIV-1 than those without HIV-1 infection. The clinical implications of sub-clinical $P$. falciparum infection among HIV-1 infected persons warrant additional study.
\end{abstract}

Keywords: Malaria, P. falciparum, HIV-1

\section{Background}

Malaria and HIV each cause substantial morbidity and mortality in sub-Saharan Africa, where they extensively overlap geographically. It is estimated that in 2009 approximately 2.5 million deaths were attributable to malaria and/or HIV-1 infection [1,2]. Although it was initially thought that the two epidemics were

\footnotetext{
* Correspondence: rschooley@ucsd.edu

2Department of Medicine, University of California, San Diego, San Diego, CA, USA

${ }^{5}$ Current address: Division of Infectious Diseases, University of California, San Diego, Mail Stop 0711, 9500 Gilman Dr, La Jolla, CA 92093, USA Full list of author information is available at the end of the article
}

independent, more recent studies demonstrate that these two pathogens negatively impact each other from several perspectives [3-5]. During clinically apparent bouts of Plasmodium falciparum infection, plasma HIV RNA levels increase [6]. $\mathrm{CD}_{4}^{+} \mathrm{T}$ lymphocytes decline temporarily during clinical malaria episodes in both HIVinfected and uninfected patients [7]. Repeated malaria infections are associated with a more rapid decline in $\mathrm{CD}^{+} \mathrm{T}$ lymphocyte counts over time in HIV-1 infected individuals. These findings suggest that symptomatic $P$. falciparum infections may accelerate progression of HIV disease [7]. Conversely, recent studies indicate that 
persons with HIV-1 infection, especially those with more advanced disease, are at greater risk for clinical malaria $[8,9]$. In addition, bouts of clinical malaria in HIVinfected patients, are more likely to be associated with increased morbidity and mortality and higher relapse rates following therapy [10].

Over the past several years, Mozambique's national malaria control programme has increased its efforts to reduce morbidity and mortality from malaria. These efforts have included intra-domiciliary spraying, the distribution of impregnated bed nets (especially for pregnant women and children below five years of age), and intermittent chemoprophylaxis in pregnant women. These measures have contributed to reductions in malaria-related morbidity and mortality, especially in urban areas of the country. Clinical cases of malaria reported to Mozambique's Ministério da Saúde (Ministry of Health) declined from 6,155,082 in 2007 to 4,091,614 by 2009 . Also, deaths attributed to malaria decreased from 3,889 to 2,311 over the same time interval $[11,12]$. Despite these reductions, malaria remains a major cause of morbidity and mortality in Mozambique, especially among women and children, and still accounts for $44 \%$ of outpatient visits, $57 \%$ of pediatric admissions and $26 \%$ of all hospital admissions [13].

Mozambique also ranks among the top tier of countries in HIV-1 with an estimated adult prevalence rate of 14\% (in those 15-49 years of age)[14]. Mozambique, therefore, serves as an excellent venue in which to further delineate interactions between these two pathogens. The prevalence of sub-clinical malaria (which is defined as having microbiological evidence of infection without any clinical symptoms) was studied in two locations in Mozambique. This study was conducted in an effort to better understand the prevalence of subclinical $P$. falciparum parasitaemia in two representative locations within Mozambique by blood smear and by polymerase chain reaction (PCR) technology. In addition, the study examined whether sub-clinical parasitaemia was affected by HIV-1 infection and/or trimethoprim-sulphamethoxazole (TS) prophylaxis.

\section{Methods}

\section{Study design}

This was a prospective cross-sectional study designed to determine the prevalence of sub-clinical malaria in HIV1 infected and uninfected control volunteers residing in similar locations. In addition, relationships among malarial parasitaemia, HIV status and anti-malarial intervention efforts were examined.

\section{Study sites and populations sampled}

Study participants were recruited from two hospitals in Maputo (the Polana Caniço Hospital and the Maputo
Military Hospital) and from the Mocuba District Hospital, which served as a representative rural location in Zambeze province.

\section{Enrollment procedures and sample collection}

Volunteers who were at least 18 years of age and seeking care or voluntary testing for HIV-1 were recruited for this study. Prior to enrollment, a nurse or HIV counselor explained the study to participants and invited their participation. Volunteers who agreed to participate and provided written informed consent were enrolled into the study. The National Bioethics Committee of Mozambique and the Human Research Protections Program of the University of California, San Diego, approved the study. Demographic and clinical data including gender, age, CD4 cell count, HIV-1 treatment history and the use of selected concomitant medications (including trimethoprim-sulphamethoxazole [TS] were recorded. Patients were also asked if they had received therapy for malaria and, if so, how recently and what drug was administered. Capillary blood was obtained by finger stick from those seeking HIV testing. One spot of blood was put onto a slide, stained with Giemsa and examined by optical microscopy [15]. Approximately fifty uL of blood was also placed on Whatman \# 2 filter paper and allowed to air dry. The blood spot was then fixed in methanol and air dried prior to storage at $4^{\circ} \mathrm{C}$ until shipment to San Diego, where they were stored at $-20^{\circ} \mathrm{C}$ until processing for PCR.

\section{Detection of $P$. falciparum DNA by PCR}

Half of each blood spot was used to extract DNA using a saponin/chelex extraction. Briefly, the DBS was placed in $0.5 \%$ saponin in PBS over night at $4^{\circ} \mathrm{C}$. The next day the saponin solution was aspirated and replaced with $1 \mathrm{~mL}$ of PBS and placed at $4^{\circ} \mathrm{C}$ for 15-30 minutes. The DBS was then placed into pre-heated 5\% Chelex water solution, vortexed and incubated for 10 minutes at $100^{\circ} \mathrm{C}$. Vortexing was repeated five minutes through the incubation, and at the end of the incubation. Tweezers were used for all transfers of the DBS between tubes and were flamed between each transfer. The DBS/Chelex solution was then centrifuged at $10,000 \mathrm{~g}$ for $2 \mathrm{~min}$ and the supernatant was moved to a new tube and was either used for PCR or stored at $-20^{\circ} \mathrm{C}$.

The PCR for P. falciparum detects a 700 bp portion of the Merozoite Specific Protein 2 ( $m s p 2)$ gene. Reactions were performed using $2 \mu \mathrm{L}$ of supernatant from the DBS DNA extraction in a $50 \mu \mathrm{L}$ PCR reaction $(2.5 \mathrm{U}$ Taq polymerase [Invitrogen], $3 \mathrm{mM} \mathrm{MgCl} 2,500 \mathrm{nM}$ dNTPs [Fermentas], and $50 \mathrm{pmol}$ of each primer [IDT]). In the first round of the nested PCR, the gene was amplified using primers msp2_1F (5'-GAAGGTAATTAAAACATTGTC-3') 
and $m s p 2$ 1R (5'-GAGGGATGTTGCTGCTCCACAG-3'). For the PCR, denaturation was performed at $94^{\circ} \mathrm{C}$ for 5 minutes, followed by 30 cycles of denaturation at $94^{\circ} \mathrm{C}$ for 30 seconds, annealing at $55^{\circ} \mathrm{C}$ for 2 minutes, and elongation at $70^{\circ} \mathrm{C}$ for 2 minutes, with a final extension at $72^{\circ} \mathrm{C}$ for 7 minutes. $1 \mu \mathrm{L}$ of the outer PCR product was used as the template for the inner PCR reaction using primers MSP2_2F (5'-GAGTATAAGGAGAAGTATG-3') and MSP2_2R (5'-CTAGAACCATGCATATGTCC-3'), using the same reaction times and temperatures. The PCR products from the inner PCR were run on a $1 \%$ agarose gel at $175 \mathrm{~V}$ for 30 minutes and stained with ethidium bromide for visualization.

\section{Detection of malarial parasites by light microscopy}

Two independent microscopists read the smears for malaria parasites. A third microscopist who was blinded to the original results examined slides with discordant results and the final results of the parasitaemia were the average of the two similar counts. Results were expressed as the number of visible parasites/ $\mu \mathrm{L}$ blood.

\section{HIV testing}

HIV testing was performed in parallel with two rapid HIV antibody detection kits (Determine [Abbott Laboratories] and Unigold [Trinity Biotech]).

\section{Data analysis}

Data were analysed using EPI INFO (version 3.4.3-CDCAtlanta). Continuous variables were compared using Student's $\mathrm{t}$ tests for variables not normally distributed. Proportions were compared using the $X^{2}$ test or the Fisher's exact test. A 2-tailed $P$ value of $\leq 0.05$ was judged to be significant. Univariate and stratified analyses of data on age, sex, parasite density, and HIV serostatus, use of TS, antiretroviral therapy regimen (ART) and fever, CD4 cell count were conducted to identify potential confounders and effect modifiers of the associations between the two pathogens. Statistical measure of inter-rater reliability was calculated using Fleiss' kappa index formula $[16,17]$.

\section{Results}

\section{Study population demographics}

The algorithm shown in Figure 1 presents the overall screening for HIV and malaria testing of the entire study population. Overall 1,835 persons were recruited (Maputo, $\mathrm{n}=1,115$, and Mocuba $\mathrm{n}=720$ ) of whom 1,280 (69.8\%) were HIV infected and 555 (30.2\%) were HIV seronegative. The mean age of the study population was 32.6 years (range, 18 - 79) for the HIV infected cohort and 30.7 years (range, 18 - 66) for the uninfected cohort. The HIV infected cohort was $64 \%$ female and 36\% male.
Table 1 summarizes the prevalence of $P$. falciparum by microscopy and the prevalence of $P$. falciparum DNA by nested PCR by location. A greater proportion of those recruited in Maputo (78.6\%) than Mocuba (56.1\%) were HIV-1 infected.

Blood smears from all 1,835 study participants were examined by light microscopy for malarial parasites and the results are presented in Table 1. PCR for P. falciparum DNA was performed on a subset of 1249 (68.1\%) of the study population. The overall prevalence of malaria by microscopy was higher in Mocuba than in Maputo $(5.1 \%$ vs. $0.4 \%, \mathrm{p}<0.001)$. Likewise P.m falciparum DNA was detected more frequently in Mocuba (53 of 387 study participants, $14.7 \%$ ) than in Maputo (16 of 862 study participants $(1.9 \%)(\mathrm{p}=0.001)$.

\section{Comparison of parasitaemia rates in the HIV-1 infected and uninfected populations}

In Mocuba, blood smear positivity for P. falciparum was significantly higher among HIV infected individuals compared to HIV uninfected individuals [39/404 (9.9\%) vs. $16 / 316(5.1 \%), \mathrm{p}<0.02$ ]. Although the same trend toward a higher prevalence of malaria in the HIV infected population than in the uninfected population was noted when assessed by PCR $[(37 / 204,18.2 \%)$ and $(20 / 183$, $10.9 \%)]$, the difference did not reach statistical significance $(\mathrm{p}<0.1)$. In Maputo only $0.5 \%$ of the 1115 study participants were smear positive for $P$. falciparum and only 16 of the $862(1.8 \%)$ had detectable P. falciparum DNA in plasma. In those with blood smears that were positive for malarial parasites, there was a trend toward a higher mean parasitaemia level in HIV-1 infected study participants $(11786 \pm 28267$ parasites/ $\mu$ l) compared to those who were not infected with HIV-1 $(2425 \pm 4701$ parasites $/ \mu \mathrm{l})(\mathrm{p}=0.08)$.

\section{Impact of TS and ART use on parasitaemia}

National HIV treatment guidelines at the time of the study recommended that HIV-1 infected persons with $<250 \mathrm{CD} 4$ cells $/ \mathrm{mm}^{3}$ be prescribed chronic TS prophylaxis. During the period of the study, however, TS was out of stock in Maputo and thus few Maputo participants received the drug. In Mocuba information about TS prophylaxis was available for 397 of the 404 participants whose blood smears were examined for parasites and from 201 of the 204 examined by PCR (Table 2). Parasitaemia rates were similar by smear 10.7 vs. $8.4 \%$ for those on TS prophylaxis compared to those not on TS respectively. Similarly, P. falciparum DNA was detected at a similar rate in the two groups (14.3 vs. $17.9 \%)$ respectively. Information about antiretroviral therapy status and malarial positivity was available for 335 participants from Mocuba. Parasitaemia rates were similar between those who reported receiving 


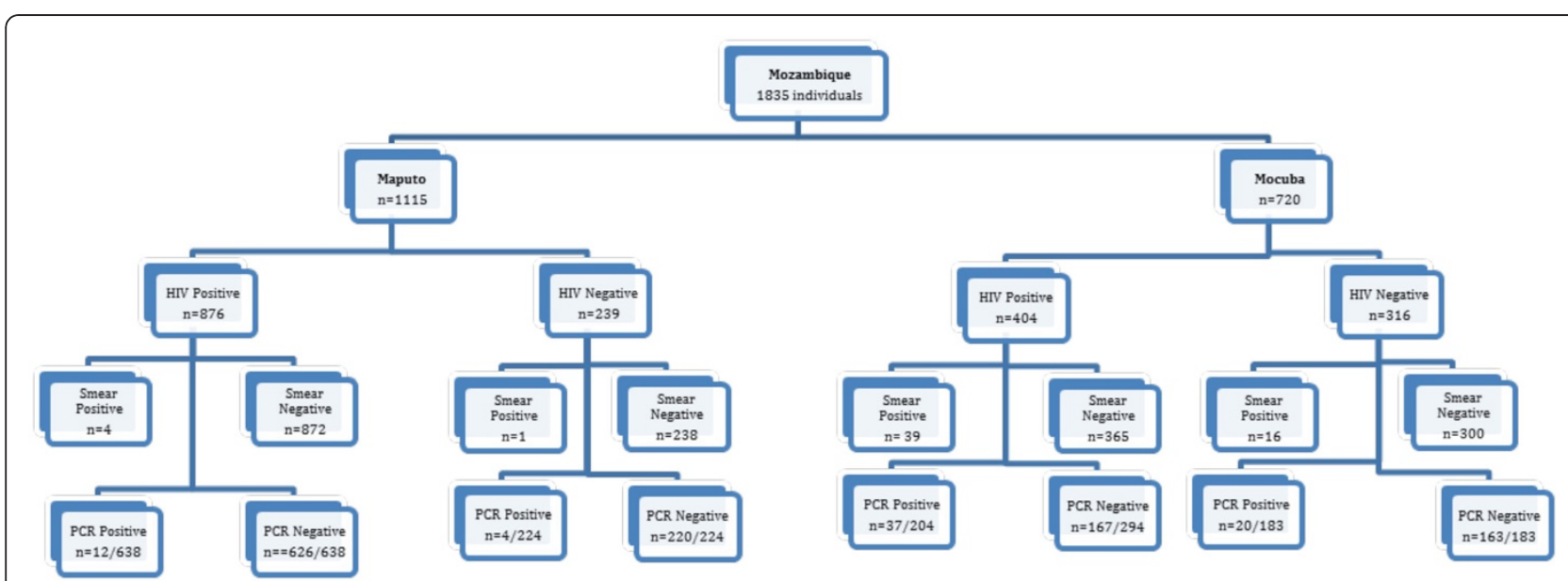

Figure 1 - Testing algorithm for study participants.

antiretroviral drugs $(9 / 87,10.3 \%)$ and those not reporting use of antiretroviral drugs $(30 / 248,12 \%)$.

\section{Comparison of the sensitivity and Kappa index of light} microscopy and PCR

Using visual inspection of the peripheral blood smear as the gold standard reference for malaria parasite detection, the sensitivity and specificity of PCR is presented in Table 3. There is a substantial agreement between the smear and PCR for detection of malaria $k=0.646$ [0.543-0.748]. Indeed, the efficiency of PCR for a positive or negative result is $97 \%$. Overall, PCR detected malarial parasitaemia in 73 of 1249 (5.8\%) study participants compared to 60 of 1835 (3.3\%) participants

Table 1 Frequency of positivity for Plasmodium falciparum by direct smear examination or of $P$. falciparum DNA by nested PCR by location

\begin{tabular}{|c|c|c|c|c|c|c|c|}
\hline & & \multicolumn{2}{|c|}{ Maputo $n=1115$} & \multicolumn{2}{|c|}{ Mocuba $n=720$} & \multicolumn{2}{|c|}{ Total (both sites) $n=1835$} \\
\hline & & $n$ & $(\%)$ & $\mathrm{n}$ & (\%) & & (\%) \\
\hline HIV Positive & & 876 & $(68.0)$ & 404 & $(32.0)$ & 1280 & $(100)$ \\
\hline \multirow[t]{3}{*}{ Smear } & & 876 & & 404 & & 1280 & $(100)$ \\
\hline & Positive & 4 & $(0.5)$ & 39 & (9.6) & 43 & (3.3) \\
\hline & Negative & 872 & (99.5) & 365 & $(90.4)$ & 1237 & $(96.7)$ \\
\hline \multirow[t]{3}{*}{ PCR } & & 638 & & 204 & & 842 & $(100)$ \\
\hline & Positive & 12 & $(1.9)$ & 37 & $(18.1)$ & 49 & (5.8) \\
\hline & Negative & 626 & $(98.1)$ & 167 & $(81.9)$ & 793 & $(94.2)$ \\
\hline HIV Negative & & 239 & $(43.0)$ & 316 & (57) & 555 & $(100)$ \\
\hline \multirow[t]{3}{*}{ Smear } & & 239 & & 316 & & 555 & $(100)$ \\
\hline & Positive & 1 & $(0.4)$ & 16 & (5.1) & 17 & (3.1) \\
\hline & Negative & 238 & (99.6) & 300 & $(94.9)$ & 538 & (96.9) \\
\hline \multirow[t]{3}{*}{ PCR } & & 224 & & 183 & & 407 & (100.0) \\
\hline & Positive & 4 & $(1.8)$ & 20 & (10.9) & 24 & (5.9) \\
\hline & Negative & 220 & $(98.2)$ & 163 & (89.1) & 383 & $(94.1)$ \\
\hline \multicolumn{8}{|l|}{ Total } \\
\hline & Smear & 1115 & (61) & 720 & (39) & 1835 & $(100)$ \\
\hline & Positive & 5 & $(0.5)$ & 55 & (7.6) & 60 & (3.2) \\
\hline & Negative & 1110 & (99.6) & 665 & (92.4) & 1775 & (96.8) \\
\hline & $P C R$ & 862 & & 387 & & 1249 & (100) \\
\hline & Positive & 16 & $(1.8)$ & 53 & (13.7) & 69 & $(6.0)$ \\
\hline & Negative & 846 & (96.5) & 334 & (86.3) & 1198 & $(94.0)$ \\
\hline
\end{tabular}


Table 2 Sensitivity, specificity and efficiency of PCR to detect malaria in the study population

\begin{tabular}{llccllll}
\hline Test & & \multicolumn{2}{c}{ Smear } & Total & Sen. & Spec. & Eff. \\
\cline { 2 - 4 } \cline { 5 - 6 } & & Positive & Negative & & & & \\
\hline PCR & Positive & 38 & 35 & 73 & 0.90 & 0.97 & 0.97 \\
& Negative & 4 & 1,172 & 1,176 & & & \\
\multirow{2}{*}{ Total } & 42 & 1,207 & 1,249 & & & \\
\hline
\end{tabular}

Data represent the number of positive and negative individuals results for samples tested by both methods. Sen = Sensitivity, Spec = specifcity and $\mathrm{Eff}=$ efficiency. Data are the proportion of true positive and true negative (using the peripheral blood smear as the gold standard test).

evaluated by light microscopy $\left(X^{2}=10.9, \mathrm{p}<0.01\right)$. This difference in sensitivity was driven by the HIV-1 infected population where the rate of parasitaemia was $49 / 842$ (5.8\%) as assessed by PCR but only 43/1,280(3.4\%) when assessed by microscopy. By contrast, the rates of parasitaemia were comparable $(\sim 3 \%)$ in the HIV-1 uninfected population whether assessed by microscopy or PCR. Plasmodium falciparum DNA was detected by PCR in 38 of the $42(90.4 \%)$ samples that were positive by microscopy. In contrast, parasites were detected by microscopy in only 38 of the 73 (52\%) of those that had $P$. falciparum DNA detected by PCR analysis.

\section{Discussion}

In this study, sub-clinical malaria was more prevalent in HIV-1 infected individuals than in HIV-1 seronegative populations when assayed by either smear or nested DNA PCR. Sub-clinical parasitaemia was less frequent in Maputo where malaria eradication efforts have been more intensive over the past several years in comparison with rural areas of the country. The significantly higher rate of sub-clinical malaria in asymptomatic individuals from Mocuba and the use of TS prophylaxis in HIV-1 infected persons with lower CD4 cell counts provided an opportunity to evaluate the impact of TS prophylaxis on the prevalence of sub-clinical malaria by both light microscopy and PCR. Although the practice of prescribing TS prophylaxis primarily to those with lower CD4 cell counts was a potential confounding factor, our data

Table 3 Effect of trimethoprim-sulphamethoxazole prophylaxis on malarial positivity by direct smear examination and PCR among participants enrolled in Mocuba

\begin{tabular}{lccll}
\hline & On TS & Not on TS & TS Status Unknown & Total \\
\hline Smear Positive & $3(10.7 \%)$ & $31(8.4 \%)$ & $5(71.4 \%)$ & 39 \\
Smear Negative & $25(89.3 \%)$ & $338(91.6 \%)$ & $2(28.6 \%)$ & 365 \\
Total & 28 & 369 & 7 & 404 \\
PCR Positive & $4(14.3 \%)$ & $31(17.9 \%)$ & $2(66.7 \%)$ & 37 \\
PCR Negative & $24(85.7 \%)$ & $142(82.1 \%)$ & $1(33 \%)$ & 167 \\
Total & 28 & 173 & 3 & 204 \\
\hline
\end{tabular}

indicate that TS prophylaxis does not eliminate subclinical parasitaemia whether assessed by microscopy or PCR. Among those who were smear positive for malaria, more than twofold higher parasite density levels were observed in the HIV-1 infected population than among those who were HIV-1 seronegative, in contrast to what has been previously reported [18]. Although both populations were recruited from people seeking care from HIV-1 testing and treatment sites in the same clinical facilities, caution in interpreting the differences in prevalence and parasitaemia levels between the HIV-1 infected and HIV-1 seronegative populations is warranted since other differences in the two populations might be unrecognized.

It is also possible that the observed differences in prevalence and levels of parasitaemia between the HIV-1 infected and uninfected populations might actually underestimate the effect of HIV-1 infection on malarial parasitaemia rates since HIV-1 infected individuals since those under care for HIV might be more likely to be in contact with the health care system and have more access to bed nets and other preventive measures. Furthermore, since syndromic treatment of fever with empiric anti-malarial therapy is not uncommon, persons with fever from HIV-1 or its complications could be more likely to receive empiric intermittent anti-malarial therapy for other disease states which might further lower the prevalence of sub-clinical malaria in this group. An additional limitation of the study was that HIV RNA levels are not routinely monitored in Mozambique and, thus, viral load data were not available.

TS prophylaxis in HIV patients reduces morbidity and mortality from malaria as well as from Pneumocystis jirovecii $[19,20]$. In view of the low prevalence of malarial parasitaemia and of clinical malaria in Maputo, TS would seem to be less critical from the perspective of reducing morbidity and mortality from malaria than in places like Mocuba, where the risk of malaria remains higher. The potential impact of TS on other HIV-1 related opportunistic infections, such as Pneumocystis jirovecii pneumonia would, nonetheless, continue to be important. It is well demonstrated that HIV infection predisposes to more frequent and more severe episodes of malaria $[3,5,9,10]$. Since this was a cross-sectional rather than a longitudinal study, time-dependent malariarelated morbidity and mortality rates were beyond the scope of this study. Nonetheless, in Mocuba where $P$. falciparum elimination efforts have been less successful than in Maputo, it was found that the HIV cohort had 1.7 times more sub-clinical malaria than the HIV uninfected cohort as defined by PCR. Others have demonstrated that overt clinical bouts of malaria are associated with increased levels of HIV-1 RNA and of CD4 cell decline $[6,21]$. The implications of sub-clinical malarial 
parasitaemia for HIV-1 disease progression have not yet been delineated, but it would seem that such studies are warranted [22,23]. Finally, although the impact of subclinical malarial parasitaemia on the risk of clinical malaria in either the HIV-1 infected or the HIV-1 seronegative population or on the progression of HIV-1 in the co-infected population has not been fully delineated, the sensitivity of nested PCR of dried blood spots for detecting carriage of $P$. falciparum might make this an attractive approach to monitor the success or failure of malaria control efforts in areas, such as Mocuba, as more concerted efforts to eliminate malaria are undertaken.

\section{Competing interests}

Dr. Schooley is a member of the Scientific Advisory Board of Gilead Sciences.

\section{Authors' contributions}

EVN and RTS designed, obtained research funding and were responsible for the overall conduct of the study. RB and VR contributed to data analysis and preparation of the manuscript. BP, CG and MO contributed to study design and analysis and were responsible for sample collection and conducted the PCR assays. All authors read and approved the final manuscript.

\section{Acknowledgments}

We express our gratitude to all patients who contributed to this study. We also acknowledge to the staff of the health units where the study was done. Special thanks go to Drs. Carlos Funzamo and Antonio Junior for their contributions in organizing the logistics for patient recruitment and sample shipment from Mocuba to Faculty of Medicine, Eduardo Mondlane University, and Maputo. The study was funded by the US National Institutes of Health through an International Pilot Grant from the UCSD Center for AIDS Research, (5P30AI036214-15) and by a grant from the Gilead Foundation

\section{Author details}

1Department of Microbiology, Universidade Eduardo Mondlane, Maputo, Mozambique. ${ }^{2}$ Department of Medicine, University of California, San Diego, San Diego, CA, USA. ${ }^{3}$ Instituto Higiene e Medicina Tropical, Universidade Nova de Lisboa, Lisbon, Portugal. ${ }^{4}$ Department of Medicine, Universidade Federal da Bahia, Salvador, Brazil. ${ }^{5}$ Current address: Division of Infectious Diseases, University of California, San Diego, Mail Stop 0711, 9500 Gilman Dr, La Jolla, CA 92093, USA.

Received: 22 December 2011 Accepted: 8 June 2012

Published: 1 August 2012

\section{References}

1. UNAIDS Global reports: UNAIDS report on the Global AIDS epidemic 2010. WHO library Cataloguing-in-Publication Data JC1958E. 2010.

2. World Health Organization: World malaria report 2010. Geneva: WHO/HTM/ GMP/2010.1; 2010 .

3. Cohen C, Karstaedt A, Frean J, Thomas J, Govender N, Prentice E, Dini L, Galpin J, Crewe-Brown H: Increased prevalence of severe malaria in HIVinfected adults in South Africa. Clin Infect Dis 2005, 41:1631-1637.

4. Cuadros DF, Branscum AJ, Crowley PH: HIV-malaria co-infection: effects of malaria on the prevalence of HIV in East sub-Saharan Africa. Int J Epidemiol 2011, 40:931-939.

5. Otieno RO, Ouma C, Ong'echa JM, Keller CC, Were T, Waindi EN, Michaels MG, Day RD, Vulule JM, Perkins DJ: Increased severe anemia in HIV-1exposed and HIV-1-positive infants and children during acute malaria. AIDS 2006, 20:275-280.

6. Kublin JG, Patnaik P, Jere CS, Miller WC, Hoffman IF, Chimbiya N, Pendame R, Taylor TE, Molyneux ME: Effect of Plasmodium falciparum malaria on concentration of HIV-1-RNA in the blood of adults in rural Malawi: a prospective cohort study. Lancet 2005, 365:233-240.

7. Mermin J, Lule JR, Ekwaru JP: Association between malaria and CD4 cell count decline among persons with HIV. J Acquir Immune Defic Syndr 2006, 4:129-130.
8. French N, Nakiyingi J, Lugada E, Watera C, Whitworth JA, Gilks CF: Increasing rates of malarial fever with deteriorating immune status in HIV-1-infected Ugandan adults. AIDS 2001, 15:899-906.

9. Whitworth J, Morgan D, Quigley M, Smith A, Mayanja B, Eotu H, Omoding N, Okongo M, Malamba S, Ojwiya A: Effect of HIV-1 and increasing immunosuppression on malaria parasitaemia and clinical episodes in adults in rural Uganda: a cohort study. Lancet 2000, 356:1051-1056.

10. Grimwade K, French N, Mbatha DD, Zungu DD, Dedicoat M, Gilks CF: HIV infection as a cofactor for severe falciparum malaria in adults living in a region of unstable malaria transmission in South Africa. AIDS 2004, 18:547-554.

11. Ministério da Saúde: Report 2009: Programa Nacional de Controle da Malaria. Moçambique: Direcção Nacional de Saúde, Ministério da Saúde. 2009.

12. Ministério da Saúde: Report 2010, Programa Nacional de Controle da Malaria. Moçambique: Direcção Nacional de Saúde, Ministério da Saúde; 2010.

13. Ministério da Saúde: Programa Nacional de Controle da Malaria. Moçambique: 2012. www.misau.gov.mz/pt/programas/malaria.

14. Instituto Nacional de Estatística (INE): Impacto demográfico do HIV/SIDA. 2011. http://www.ine.gov.mz/hiv/ipcthivmz.

15. Gilles HM: Diagnostic methods in malaria. In HM Gilles and DA Warrell. Bruce Chwatt's Essential Malariology. 3rd edition.; 1993:79-95.

16. Cohen J: Weighted kappa: nominal scale agreement with provision for scaled disagreement or partial credit. Psychol Bull 1968, 70:213-220.

17. Fleiss JL: Measuring nominal scale agreement among many raters. Psychol Bull 1971, 76:378-382.

18. Ter Kuile FO, Parise ME, Verhoeff FH, Udhayakumar V, Newman RD, van Eijk AM, Rogerson SJ, Steketee RW: The burden of co-infection with human immunodeficiency virus type 1 and malaria in pregnant women in subSaharan Africa. Am J Trop Med Hyg 2004, 71(2 Suppl):41-54.

19. Malamba SS, Mermin J, Reingold A, Lule JR, Downing R, Ransom R, Kigozi A, Hunt BM, Hubbard A, Rosenthal PJ, Dorsey G: Effect of cotrimoxazole prophylaxis taken by human immunodeficiency virus (HIV)-infected persons on the selection of sulfadoxine-pyrimethamine-resistant malaria parasites among HIV-uninfected household members. Am J Trop Med Hyg 2006, 75:375-380.

20. Thera MA, Sehdev PS, Coulibaly D, Traore K, Garba MN, Cissoko Y, Kone A, Guindo A, Dicko A, Beavogui AH, Djimde AA, Lyke KE, Diallo DA, Doumbo OK, Plowe CV: Impact of trimethoprim-sulfamethoxazole prophylaxis on falciparum malaria infection and disease. J Infect Dis 2005, 192:1823-1829.

21. Franke MF, Spiegelman D, Ezeamama A, Aboud S, Msamanga Gl, Mehta S, Fawzi WW: Malaria parasitemia and CD4 T cell count, viral load, and adverse HIV outcomes among HIV-infected pregnant women in Tanzania. Am J Trop Med Hyg 2010, 82:556-562.

22. Brouwer KC, Mirel LB, Yang C, Lal RB, Kolczak MS, Van Eijk AM, Ayisi J, Otieno JA, Nahlen BL, Steketee R, Shi YP, Lal AA: Subclinical Plasmodium falciparum infection and HIV-1 viral load. Emerg Infect Dis 2007, 13:351-353.

23. Martin-Blondel G, Soumah M, Camara B, Chabrol A, Porte L, Delobel P, Cuzin L, Berry A, Massip P, Marchou B: [Impact of malaria on HIV infection](in French). Med Mal Infect 2010, 40:256-267.

doi:10.1186/1475-2875-11-252

Cite this article as: Noormahomed et al:: A cross-sectional study of subclinical Plasmodium falciparum infection in HIV-1 infected and uninfected populations in Mozambique, South-Eastern Africa. Malaria Journal 2012 11:252. 